Bangladesh J. Plant Taxon. 15(2): 89-106, 2008 (December)

(C) 2008 Bangladesh Association of Plant Taxonomists

\title{
NINE NEW TAXA AND A NEW COMBINATION IN LAURACEAE FROM INDIA AND MYANMAR
}

\author{
M. GANGOPADHYAY ${ }^{1}$ \\ Central Botanical Laboratory, P.O. Botanic Garden, Howrah 711 103, India \\ Keywords: New taxa, Actinodaphne, Beilschmiedia, Cinnamomum, Cryptocarya, New combination, \\ Potameia tirunelvelica, Lauraceae
}

\begin{abstract}
Nine new taxa belonging to the genera Actinodaphne Nees, Beilschmiedia Nees, Cinnamomum Schaeff. and Cryptocarya R. Br. of the family Lauraceae are described and illustrated from India and Myanmar. One new combination of the family has also been appended.
\end{abstract}

\section{Introduction}

During the course of a taxonomic study of the family Lauraceae of India and its adjoining countries the author came across some interesting specimens of Actinodaphne Nees, Beilschmiedia Nees, Cinnamomum Schaeff. and Cryptocarya R. Br. deposited at CAL and PBL which on critical studies were found to be of nine new taxa. They are described and illustrated here. Beilschmiedia tirunelvelica Manickam et al. is transferred to the genus Potameia A. Thouars.

\section{New taxa}

1. Actinodaphne andamanica M. Gangop., sp. nov.

(Plate 1)

Actinodaphne sesquipedalis Hook.f. et Thomson ex Meisn. affinis, sed differt foliis latis obovatis non nitidis, basi acutis, fructus cupula nitida, fructibus parvioribus globosis.

Holotypus: Dhani reef creek, 29.?.1890, King's collector s.n. (CAL); paratypus: Namunaghar, 28.6.1890, King's collector s.n. (CAL).

Allied to A. sesquipedalis Hook.f. \& Thomson ex Meisn., but differs in having broad, obovate, non-glossy leaves which are acute at base, smooth fruit-cups and smaller globose fruits.

Branchlets terete, 5-9 $\mathrm{mm}$ in diameter, scales scarred, densely rufus-tomentose, gradually slender, compressed, with shallow longitudinal grooves at upper portion. Bud scales (lowermost) ovate, c $2 \times 3 \mathrm{~mm}$, apiculate, puberulous above, upper one deciduous. Leaves in whorls of 3 , coriaceous, dark greenish-brown, glabrous above, reddish-brown or brown, tomentose on main veins beneath, obovate or elliptic, 21-38 $\times 7.2-15.0 \mathrm{~cm}$, sub-equally acute at base, entire, strongly incurved along margin, acuminate (acumens

${ }^{1}$ E-mail: mgangopadhyay55@rediffmail.com 
18-40 mm long, acute at tip) at apex; midvein penninerved, with slight impression or flat and canaliculate above, stout, raised and quadrangular beneath; lateral nerves 8-10 pairs, oblique, distant, ascending-arcuate, faint above, raised and stout beneath; tertiary nerves closely parallel, faint above, prominent beneath; minor nervules obscure above, prominent beneath, reticulate; petioles $12-17 \mathrm{~mm}$ long, c $1.5 \mathrm{~mm}$ broad, flat and shallow canaliculate above, convex beneath, rufus-tomentellus. Inflorescences axillary, subsessile,

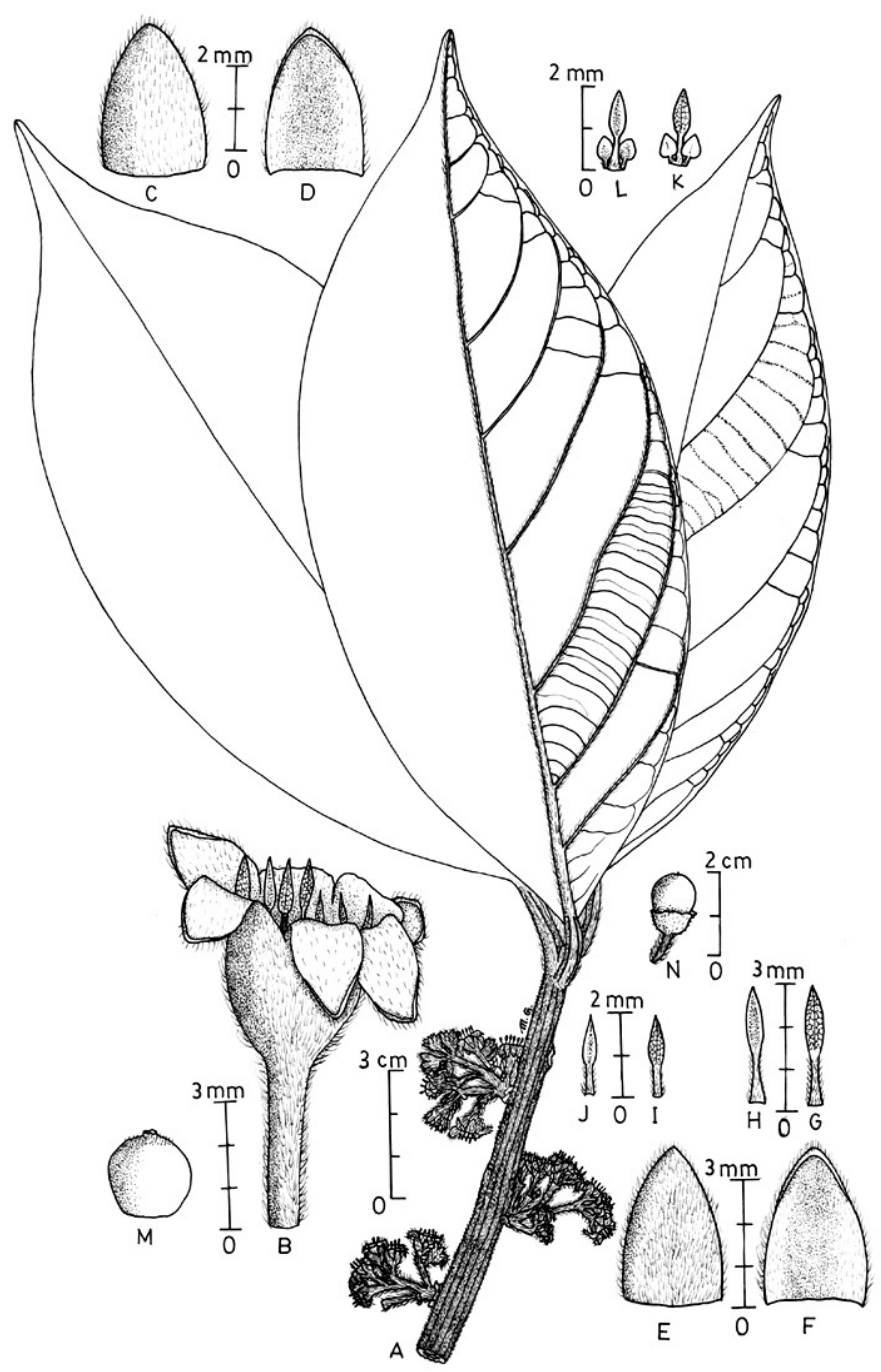

Plate 1. Actinodaphne andamanica M. Gangop. sp. nov. A. Flowering branch; B. Pistillate flower; C. Outer tepal lobe - dorsal view; D. Idem - ventral view; E. Inner tepal lobe - dorsal view; F. Idem - ventral view; G-L. Staminodes: G. Whorl I - dorsal view; H. Idem - ventral view; I. Whorl II - dorsal view; J. Idem ventral view; K. Whorl III - dorsal view; L. Idem - ventral view; M. Over-matured ovary; N. Fruit (A-M. after paratype; N. after holotype). 
panicle of umbels, 20-25 mm long, rufus-villous; peduncles short and broad, 2-3 mm long, up to $2.5 \mathrm{~mm}$ wide, bracts scarred, rufus-tomentose; branches short; bracts and bracteoles deciduous. Male flowers not seen. Female flowers (over-matured) cupularcampanulate, c $9 \times 7 \mathrm{~mm}$, villous; pedicels up to $4 \mathrm{~mm}$ long, c $1 \mathrm{~mm}$ wide. Cup oblongovoid, c $3 \times 3.5 \mathrm{~mm}$; tepal lobes $3+3$, thin, anastomosis obscure, silky pilose within; outer: ovate, c $3.5 \times 2.5 \mathrm{~mm}$, obtuse, keeled at apex; inner: ovate-lanceolate, c $3.8 \times 2.5$ $\mathrm{mm}$, subacute. Staminodes 9 in 3 whorls; whorl I: c $2.8 \mathrm{~mm}$ long; filament c $1.2 \mathrm{~mm}$ long, thin, flat, villous, head thin, narrow elliptic, acute, glabrous; whorl II: same as whorl I; whorl III: c $2 \mathrm{~mm}$ long; glands 2, c $0.8 \mathrm{~mm}$ long, stipe short, villous, head ovateoblong, acute; filament $0.8 \mathrm{~mm}$ long, villous; head narrow elliptic, acute, thin. Pistil: style and stigma broken; ovary globose, c $2 \times 2 \mathrm{~mm}$, puberulous above, glabrous below. Infructescences: bracts and bracteoles deciduous; peduncles c $4 \mathrm{~mm}$ long, $3 \mathrm{~mm}$ wide; branches short. Fruits globose, 8-10 mm in diameter, glabrous, black-brown, ruminate; pedicels 9-11 mm long, c $2.5 \mathrm{~mm}$ wide above, $1 \mathrm{~mm}$ wide below, tomentose; cup coriaceous, 3-4 × 8-9 mm, entire, smooth, fine puberulous. Flowering time: June. Fruiting time: Not known.

Distribution: India (Andaman Islands).

\section{Actinodaphne mansonii M. Gangop., sp. nov.}

Actinodaphne concolor Blume affinis, sed differt plantis glabris, foliis cum marginibus valde incurvis, apicibus acutis, supra nervis lateralibus elevatis, fructorum pedicellis longioribus.

Holotypus: Myanmar, Tenasserim, s.d., F.B. Manson 101 (CAL).

Allied to A. concolor Blume, but differs in having glabrous nature, leaves with strongly incurved margin and acute apex, raised lateral nerves on upper surface and long stalked fruits.

Branchlets terete, blackish-brown, up to $5 \mathrm{~mm}$ in diameter, smooth, glabrous, angular above. Scales of the terminal buds (outer fallen) ovate, c $3.5 \times 3.0 \mathrm{~mm}$, acute at apex, silky pilose. Leaves in whorls of 4 , thinly coriaceous, dark brown, glossy above, paler and subglaucous beneath, obovate, 22.3-25.5 $\times 8.0-9.3 \mathrm{~cm}$, attenuate at base, slightly decurrent to extreme base, cartilaginous, strongly incurved and semi-undulate along margin, acute at apex; midvein subconvex above, stout, raised, semiterete beneath, penninerved; lateral nerves 8-10 pairs, sometimes oblique, slightly raised above, stout, raised beneath, ascending, forming prominent loops near margin beneath; tertiary nerves faint above, prominent beneath, scalariform; minor nervules obscure above, faint to obscure beneath, fine reticulate; petioles $20-22 \mathrm{~mm}$ long, 2.5-3.0 mm wide, flat, canaliculate above, convex beneath. Flower not seen. Infructescences on short lateral branches, umbellate, short peduncled, silky villosulus. Fruits not seen; stalk c 10.0 × 1.5 
$\mathrm{mm}$, whitish pilose; cup shallow, 2.5-3.5 $\mathrm{mm}$ high, 7-8 $\mathrm{mm}$ in diameter, rigid, entire, blackish, glabrous. Flowering \& Fruiting time: Not known.



Plate 2. Actinodaphne mansonii M. Gangop. sp. nov. Fruiting branch (after holotype).

Distribution: Myanmar.

Local name: Kyese shor.

The plant differs from all the known species of the region in having glabrous nature and strong incurved leaf margin. All the mature fruits were found to be fallen off from the specimen. However, the other characters are sufficient for proposing this new species.

The species has been named after its collector Mr. F.B. Manson. 
3. Actinodaphne nicobarica M. Gangop., sp. nov.

(Plate 3)

Actinodaphne glomerata (Blume) Nees affinis, sed differt ramulis foliisque glabris, foliis infra cum nervis tertiariis scalariformibus confertis, pedicellisque brevioribus.

Holotypus: South Nicobar, 34 km East-West Road, 175 m, 22.7.1976, N.P. Balakrishnan 3939 (CAL); isotypus (PBL).

Allied to A. glomerata (Blume) Nees, but differs in having glabrous branchlets and leaves; leaves with fine close scalariform tertiary nerves beneath, and shorter pedicels.

Tree, 12-15 m high (fide collectoris). Branchlets terete, chocolate brown, 4-7 mm in diameter, old nodes with scars of scales, glabrous. Scales of the terminal buds variable; lower: ovate-orbicular, c $1.5 \times 2.5 \mathrm{~mm}$, rounded, margin ciliate; upper: ovate, up to $6 \times 6$ $\mathrm{mm}$, acute, sericeous above and villous along margin. Leaves in whorls of 8 , coriaceous, glossy, dark brown above, greenish, glaucous beneath, elliptic-oblong, 24-28 × 6-8 cm, cuneate at base, cartilaginous, slightly incurved along margin, acuminate (acumens up to $25 \mathrm{~mm}$ long, acute) at apex, glabrous; midvein flattened and canaliculate at lower halves, slightly raised along length above, raised and flat topped beneath; lateral nerves 10-12 pairs, slender, ascending, arcuate, forming faint loops along margin, flat above, prominent and raised beneath; tertiary nerves closely scalariform, obscure above, faint beneath; minor nervules faint, fine reticulate; petioles 18-25 mm long, 2.0-2.5 mm wide, flat and shallow canaliculate above, convex beneath, glabrous. Inflorescences subsessile, 15-22 mm long, pale brownish tomentose; peduncle short, c $2 \times 2 \mathrm{~mm}$, bracts scarred; rachis slender, c $1 \mathrm{~mm}$ wide; bracts obovate, c $5.5 \times 5.0 \mathrm{~mm}$, obtuse at apex, concave ventrally, densely sericeous outside, glabrous within; bracteoles ovate, c $3.0 \times 2.5 \mathrm{~mm}$, obtuse above, concave ventrally, sericeous. Male flowers (slightly immature): whitish green with purple tinge (fide collectoris), cupular, c $6.5 \times 3.0 \mathrm{~mm}$, densely sericeous above; pedicels c $3 \mathrm{~mm}$ long, 0.8-1.0 mm wide, angled; tepal lobes $3+3$, anastomosis obscure; outer: ovate-oblong to oblong, c $3.0 \times 2.8 \mathrm{~mm}$, acute at apex, thick, finely ciliate along margin near apices, glabrous within; inner: obovate-oblong, c $3 \times 2 \mathrm{~mm}$, obtuse, almost flat, margin fimbriate near apex, sericeous below within. Stamens 9 in 3 whorls, anthers 4-locular, all introrse; filament thick, flat, villous at base; whorl I: c $2.5 \mathrm{~mm}$ long; filament c $0.8 \mathrm{~mm}$ long; anther narrow oblong, bluntly subapiculate at apex; whorl II: c $2.5 \mathrm{~mm}$ long; filament c $1 \mathrm{~mm}$ long, anther broad oblong, obtuse; whorl III: c $2.8 \mathrm{~mm}$ long, biglandular; glands c $1.2 \mathrm{~mm}$ long, attached to filament; stipe c $0.5 \mathrm{~mm}$ long, thin, head oblong, obtuse at apex, quadrangular; filament c $1.1 \mathrm{~mm}$ long; anther oblong, obtuse. Pistillode minute. Fruits not seen. Flowering time: July. Fruiting time: Not known.

Distribution: India (Nicobar Islands).

Habitat: Grows in the inland forests at about $175 \mathrm{~m}$ altitude on clayey loam. 
According to the collector, the plant is common in Nicobar Islands but author could not find any other specimen from the locality, in the herbaria consulted, except the type.

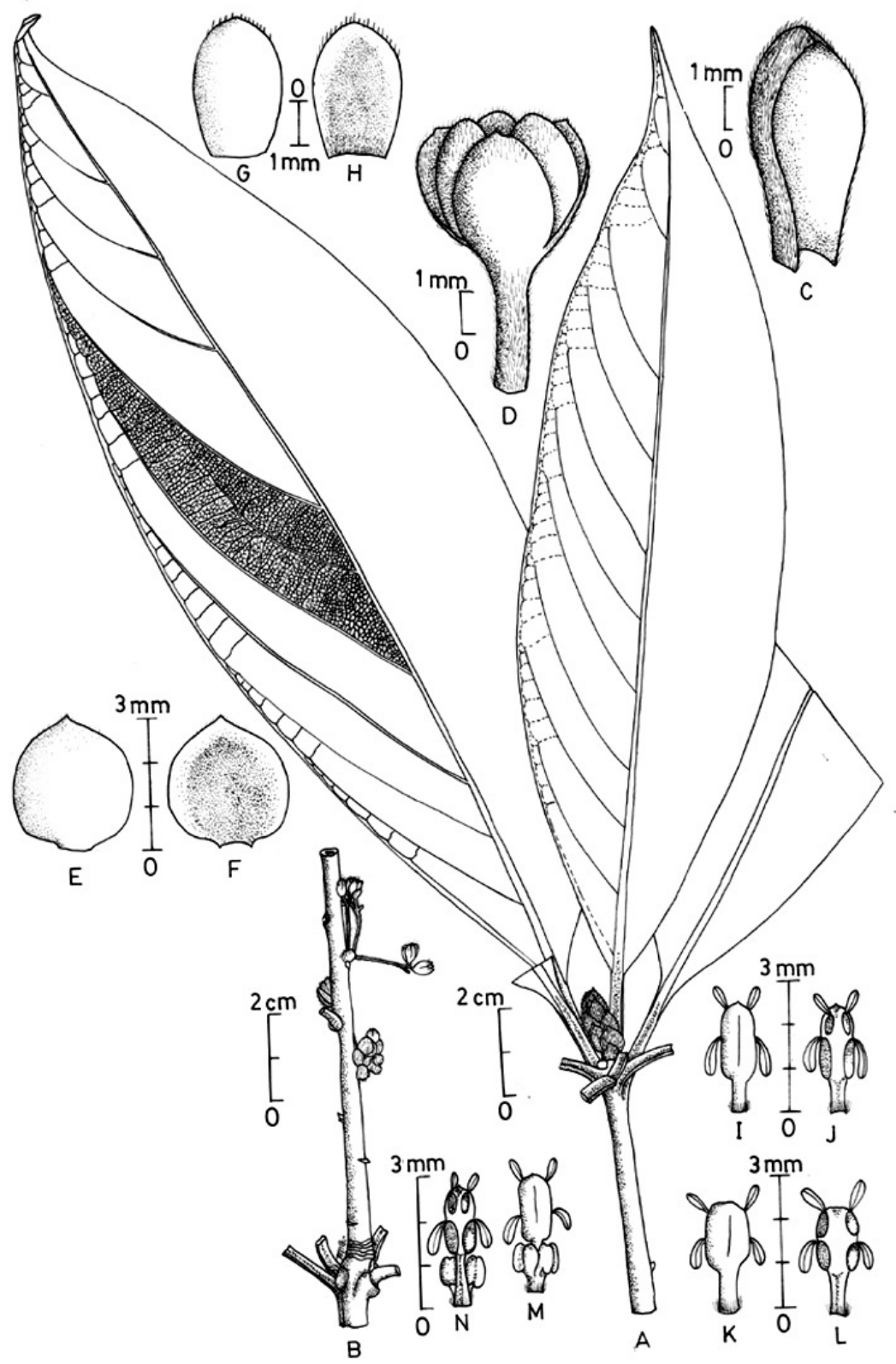

Plate 3. Actinodaphne nicobarica M. Gangop. sp. nov. A. Leafy branch; B. Inflorescence (male); C. Bract ventral view; D. Staminate flower; E. Outer tepal lobe - dorsal view; F. Idem - ventral view; G. Inner tepal lobe - dorsal view; H. Idem - ventral view; I-N. Stamens: I. Whorl I - dorsal view; J. Idem - ventral view; K. Whorl II - dorsal view; L. Idem - ventral view; M. Whorl III - dorsal view; N. Idem - ventral view (after holotype). 
4. Beilschmiedia andamanensis M. Gangop., sp. nov.

(Plate 4)

Beilschmiedia fagifolia Nees affinis, sed differt foliis rigidis, cum nervis tertiariis nervulisque minoribus obscures, fructibusque ellipsoideis acutis cum pericarpio verrucoso.

Holotypus: South Andaman, Shoal Bay, 70 m, 16.05.1990, Sam P. Mathew 20498 (PBL).

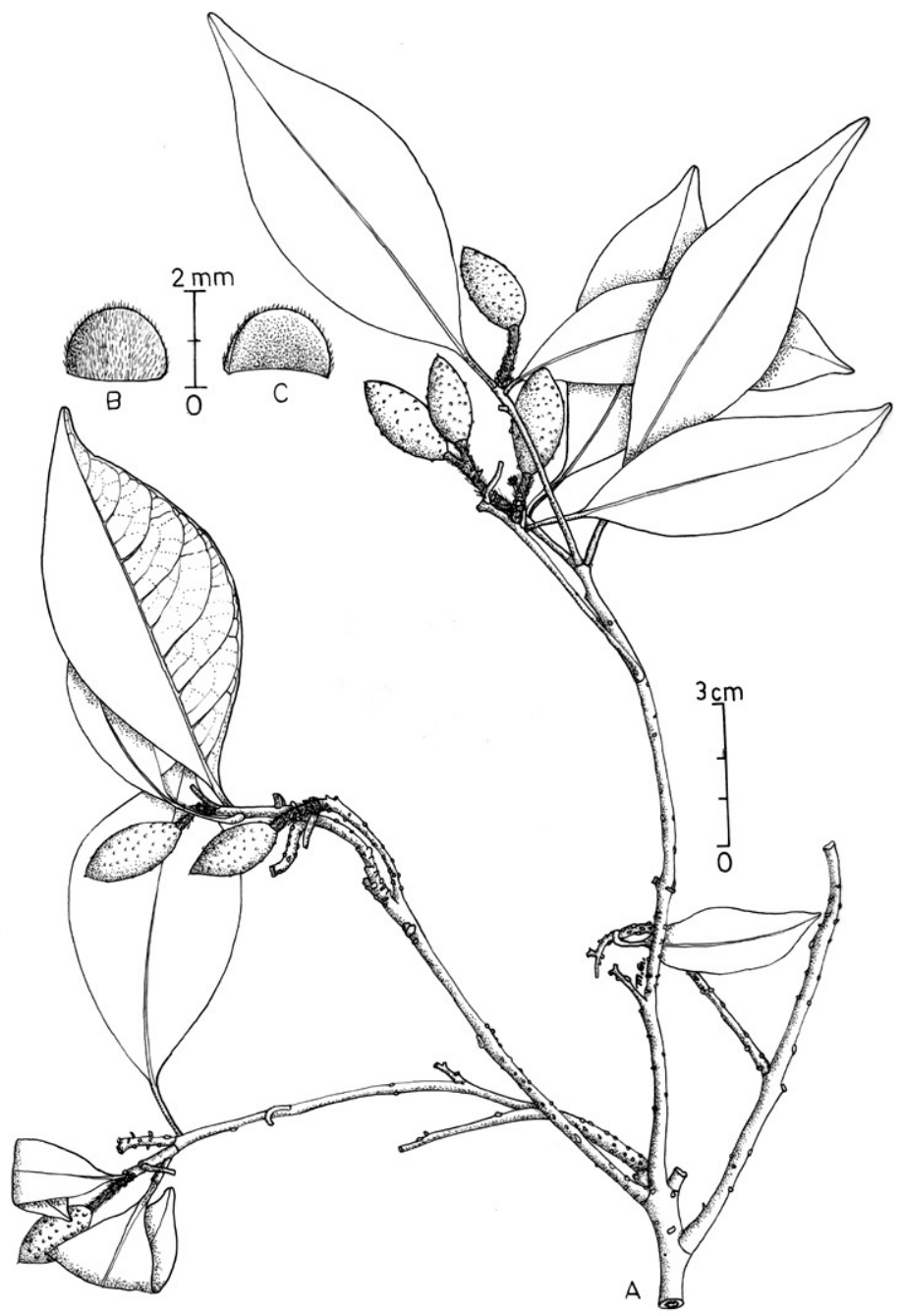

Plate 4. Beilschmiedia andamanensis M. Gangop. sp. nov. A. Fruiting branch; B. Bract - dorsal view; C. Idem - ventral view (after holotype).

Allied to B. fagifolia Nees, but differs in having stiff coriaceous leaves with entirely obscure tertiary nerves and minor nervules, and ellipsoid, acute fruits with warty pericarp. 
Tree, up to $8 \mathrm{~m}$ in height (fide collectoris). Branchlets terete, whitish grey, up to 6 $\mathrm{mm}$ in diameter, warty and lenticellate, glabrous, gradually quadrangular above. Terminal buds ovate, c $1 \times 1 \mathrm{~mm}$, acute, tomentose. Leaves opposite to sub-opposite, stiff coriaceous, greyish-green above, light brown beneath, narrow ovate to elliptic-ovate, 5$10 \times 1.5-3.8 \mathrm{~cm}$, equally cuneate to acute at base, incurved along margin, acuminate (acumens 10-11 mm long, blunt) at apex, glabrous; midvein slender, slightly raised above, flat and canaliculate beneath; lateral nerves 8-10 pairs, very slender, ascending, abruptly arcuate forming very obscure loops near margin, faint above, almost obscure beneath; tertiary nerves obscure; minor nervules obscure; petioles slender, 8-13 mm long, c $1 \mathrm{~mm}$ wide, shallowly concave above, convex beneath, glabrous. Flower not seen. Flower buds whitish (fide collectoris). Infructescences axillary, solitary, up to $3.5 \mathrm{~cm}$ long; peduncles 12-15 mm long, c $1.5 \mathrm{~mm}$ wide, terete, with scars of bracts and bracteoles; branches not present; bracts thickly coriaceous, suborbicular 1-2 × 1.0-1.5 $\mathrm{mm}$, tomentellus outside, glabrous within, deciduous. Fruits green, ellipsoid, 20-22 × 10$11 \mathrm{~mm}$, acute at apex, pericarp brown, warty, glabrous; stalks 4-6 mm long, c $1.5 \mathrm{~mm}$ wide, terete, tomentose; tepals deciduous. Flowering time: Not known. Fruiting time: May.

Distribution: India (Andaman Islands).

Habitat: Grows in the inland evergreen forests at about $70 \mathrm{~m}$ altitude.

The specimen was annotated as Dehaasia firma Blume by Dr. N.P. Singh at Kew, but it has bracteate infructescences and narrow fruit stalks, so the species certainly belongs to Beilschmiedia Nees.

5. Beilschmiedia fagifolia Nees var. gaurii M. Gangop., var. nov.

(Plate 5)

Beilschmiedia fagifolia Nees var. fagifoliae affinis, sed differt foliis rubello-brunneis nitidis, infra cum nervules laxe reticulatis, fructibusque majoribus ellipsoideis.

Holotypus: Andaman Islands, King's collector 220, acc. no. 551514 (CAL); isotypi: acc. nos. 551509-551513 \& 551515 (CAL); paratypi: South Andamans, Dhani Khari, 30.7.1892, King's collector s.n. '2 sheets' A \& B (CAL).

Allied to B. fagifolia Nees var. fagifolia, but differs in having reddish-brown, glossy leaves with laxly reticulate minor nervules beneath, and larger ellipsoid fruits.

Tree, 13-20 m high (fide collectoris). Branchlets terete, brown, up to $4 \mathrm{~mm}$ in diameter, faintly striate, glabrous, lenticellate, gradually compressed, glabrous above; terminal buds ovate-lanceolate, c $3.5 \times 2.0 \mathrm{~mm}$, brown puberulous. Leaves opposite to subopposite, sometimes alternate, throughout on branchlets, thinly coriaceous, glossy, reddish-brown above, paler beneath, elliptic, oblong-elliptic to ovate-elliptic or rarely narrow ovate, $6-16 \times 1.2-4 \mathrm{~cm}$, subequally or unequally cuneate-attenuate at base, decurrent to extreme base, entire, flat or shallowly incurved at margin, bluntly acuminate 
(acumens up to $14 \mathrm{~mm}$ long) at apex, glabrous; midvein slightly raised above, raised and flat topped beneath, slender; lateral nerves 8-10 pairs, slender, raised on both surfaces, distant, arcuate, forming weak loops with the next; tertiary nerves laxly reticulate, prominent on both surfaces, slender; minor nervules almost hidden above, faint beneath,

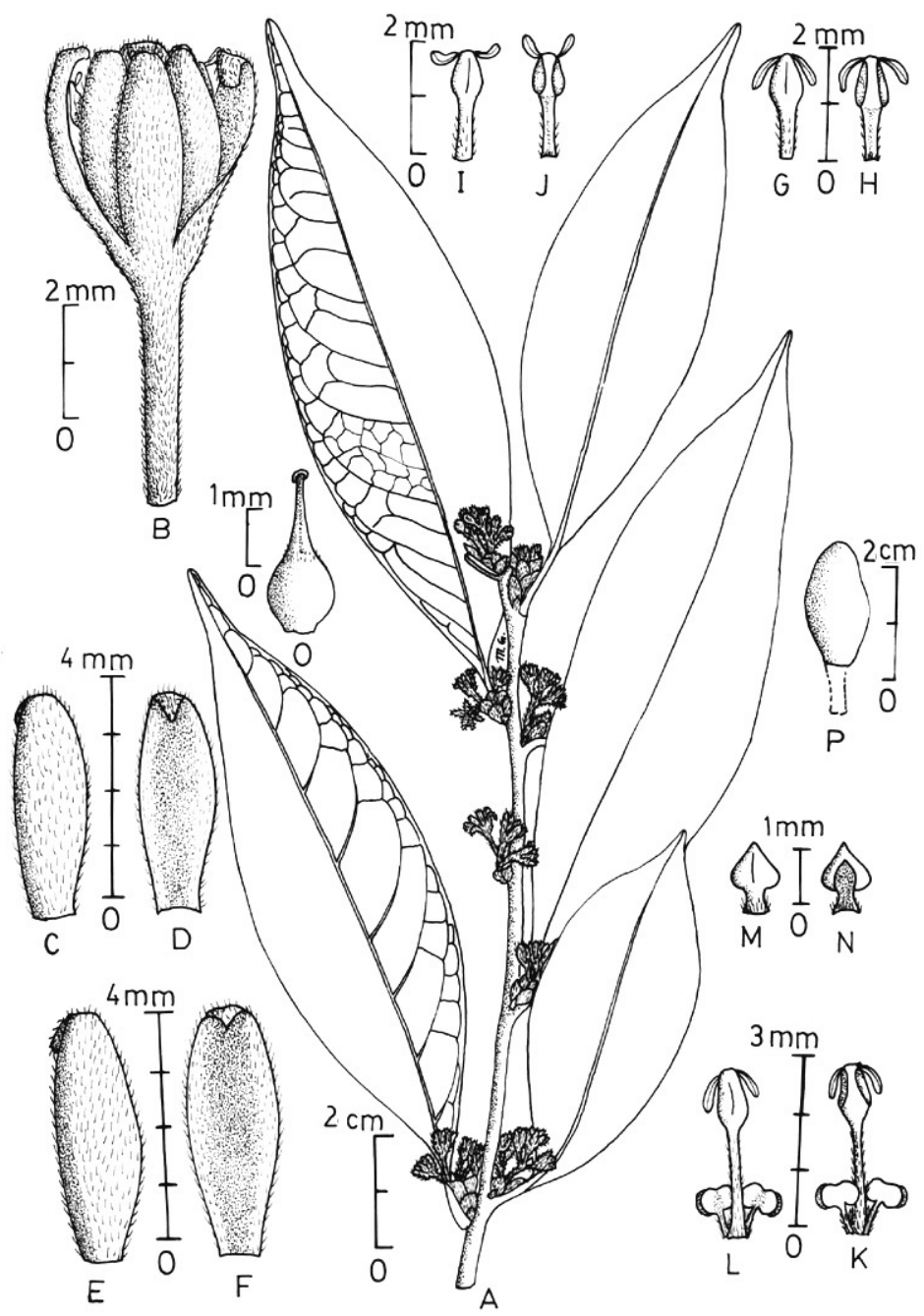

Plate 5. Beilschmiedia fagifolia Nees var. gaurii M.Gangop. var. nov. A. Flowering branch; B. Flower; C. Outer tepal lobe - dorsal view; D. Idem - ventral view; E. Inner tepal lobe - dorsal view; F. Idem - ventral view; G-N. Stamens and staminode: G. Whorl I - dorsal view; H. Idem - ventral view; I. Whorl II - dorsal view; J. Idem - ventral view; K. Whorl III - dorsal view; L. Idem - ventral view; M. Whorl IV - dorsal view; N. Idem - ventral view; O. Pistil; P. Fruit (A-O. after holotype; P. after paratype A).

reticulate; petioles $8-13 \mathrm{~mm}$ long, 0.6-1.0 mm thick, slender, concave above, convex beneath, glabrous. Inflorescences axillary, solitary, bracteate, few flowered corymbs, up to $13 \mathrm{~mm}$ long; bracts persistent, lower/outer one triangular, acute, c $1 \times 1 \mathrm{~mm}$, gradually 
ovate-orbicular above, 1.5-2.0 × 2.5-3.0 mm, obtuse, rufus-tomentellous; peduncles very short, up to $6 \mathrm{~mm}$ long; branches stout, densely rufus-villous. Flowers white (fide collectoris), shallow tubular-campanulate, c $8.5 \times 3.5 \mathrm{~mm}$, silky tomentellus; pedicels c 4 $\mathrm{mm}$ long, $0.5 \mathrm{~mm}$ wide, angled. Cup c $0.5 \times 1.2 \mathrm{~mm}$, obconic; tepal lobes $3+3$, thin, reflexed at apex, puberulous within; outer: narrow obovate-oblong, c $4.5 \times 1.2 \mathrm{~mm}$, acute, anastomosis faint; inner: ovate-oblong, c $5.0 \times 1.5 \mathrm{~mm}$, subobtuse, anastomosis obscure. Stamens 9, 3 in each whorl, anthers 2-locular; filament flat, thin, sparsely tomentellus, whorls I and II introrse, of III extrorse, biglandular; whorl I: c 1.9 mm long; filament c $1 \mathrm{~mm}$ long, wider above; anthers ovate-oblong, truncate at apex, glabrous; whorl II: c $1.9 \mathrm{~mm}$ long; filament c $1.2 \mathrm{~mm}$ long; anthers ovate, truncate and retuse at apex; whorl III: c $3 \mathrm{~mm}$ long; glands c $1.1 \mathrm{~mm}$ long, stipes thin, slender, c $0.5 \mathrm{~mm}$ long, head suborbicular, lobulate; filament c $2 \mathrm{~mm}$ long; anthers narrow-ovate, retuse at apex; whorl IV (of 3 staminodes): c $1.2 \mathrm{~mm}$ long, stipes stout, c $0.3 \mathrm{~mm}$ long, c $0.2 \mathrm{~mm}$ broad and flat, tomentellus, head triangular-ovate, acute, ventrally shallow grooved, glabrous. Pistil c $3 \mathrm{~mm}$ long, ovary globose, c $1.2 \times 1.2 \mathrm{~mm}$, smooth, glabrous, tapering towards apex; style flat, slender, shallow channeled, sparsely puberulous below; stigma thin, peltate, lobulate. Infructescences broken. Fruits ellipsoid, c $30 \times 11 \mathrm{~mm}$, subacutish toward ends, blackish; stalks c $4 \mathrm{~mm}$ long, $2 \mathrm{~mm}$ wide, fine puberulous; tepal lobes deciduous. Flowering time: Not known. Fruiting time: July.

Distribution: India (Andaman Islands).

Habitat: Grows in hill jungles on rocky places.

The fruits of the typical ones are 18-23 mm long and 9-13 mm wide.

The variety has been named in the honour of Late Gauri Roy for her dedicated service as a Librarian of Botanical Survey of India.

6. Beilschmiedia gallatlyi M. Gangop., sp. nov.

(Plate 6)

Beilschmiedia globularia Kurz affinis, sed differt gemmae terminalibus anguste ovato-lanceolatis, fructibusque parvioribus ellipsoideo-ovoideis.

Holotypus: Tenasserim, ?.1877, G.A. Gallatly 984, acc. no. 551478 (CAL); isotypi: '2 sheets': acc. nos. 551479 \& 551480 (CAL).

Allied to B. globularia Kurz, but differs in having narrow ovate-lanceolate terminal buds and smaller ellipsoid-ovoid fruits.

Tree. Branchlets terete, whitish-brown, up to $7 \mathrm{~mm}$ in diameter, rough with corky patches, glabrous, gradually smooth, slender, brown, glabrous towards apex. Terminal buds narrow ovate-lanceolate, c $6 \times 3 \mathrm{~mm}$, acute, smooth, glabrous. Leaves coriaceous, glossy, pale brown, reddish-brown to greenish-brown above, paler beneath, ovate, oblong-ovate, 10-14 × 5-6 cm, acute at base, margin entire, slightly incurved, 
subacuminate (acumens up to $6 \mathrm{~mm}$ long, blunt) at apex, glabrous; midvein slender, flat and channeled above, raised and triangular or flattened beneath; lateral nerves 8-10 pairs, distant, arcuate, prominent, forming loops at extreme margin; tertiary nerves prominent

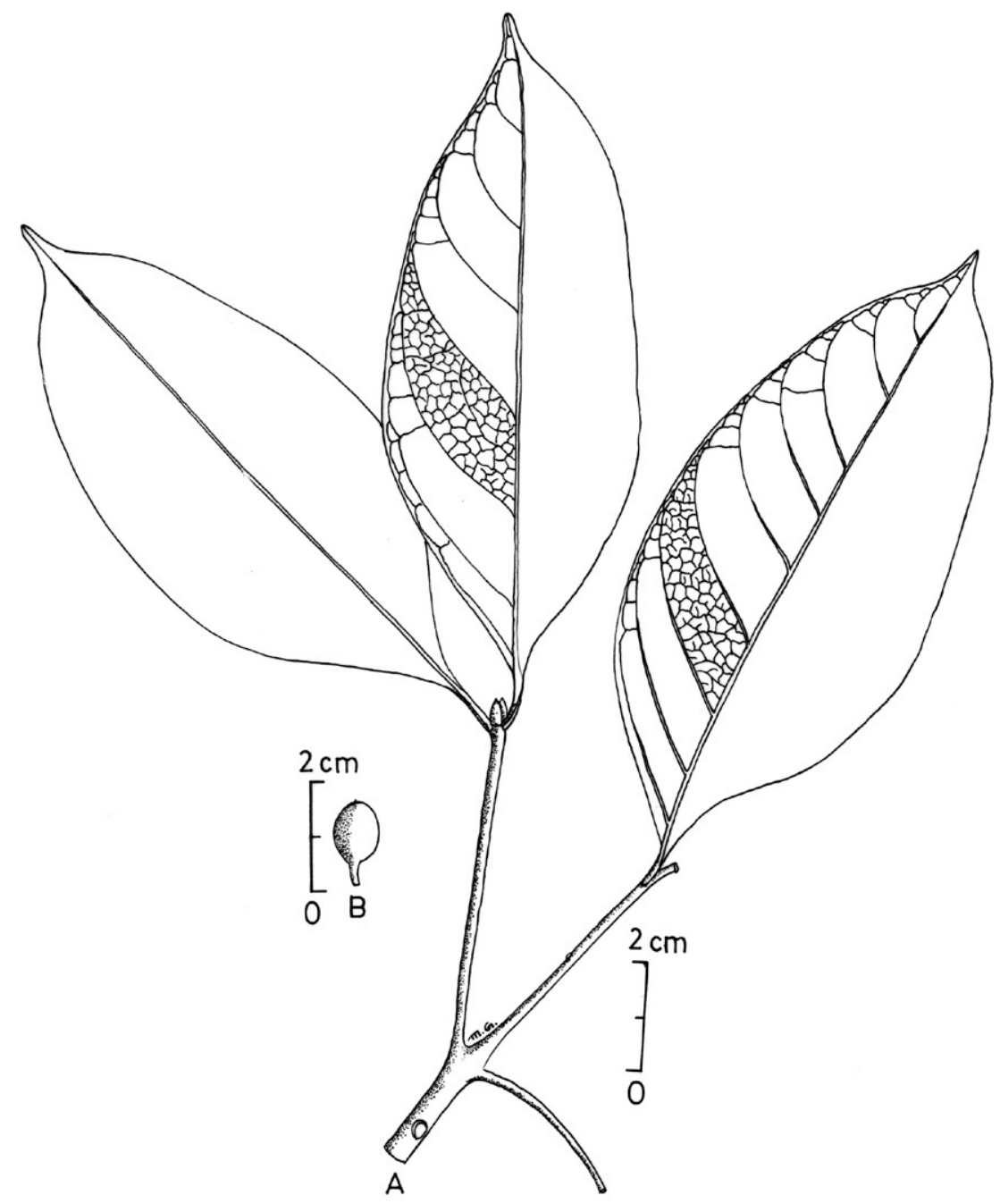

Plate 6. Beilschmiedia gallatlyi M. Gangop. sp. nov. A. Leafy branch; B. Fruit (A. after isotype acc. no. 551480; B. after holotype).

on both surfaces; minor nervules finely reticulate, more prominent beneath; petioles slender, 6-10 mm long, 1.0-1.5 mm wide, flat and concave above, terete beneath, glabrous. Flowers not seen. Infructescences up to $6 \mathrm{~cm}$ long, blackish, glabrous; peduncles terete. Fruits ellipsoid-ovoid, c $10 \times 7 \mathrm{~mm}$, obtuse with a mucro at apex, blackish, smooth, glabrous; tepals deciduous. Flowering \& Fruiting time: Not known. 
Distribution: Myanmar.

The species has been named after the well known plant collector Mr. G.A. Gallatly.

7. Cinnamomum palghatensis $M$. Gangop., sp. nov.

(Plate 7)

Cinnamomum macrocarpum Hook. f. affine, sed differt foliis angustioribus, basibus acutis, nervis secundariis confertis, fructibusque oblongis. Differt a C. walaiwarense Kosterm. foliis glabris, nervis lateralibus confertis, fructibusque oblongis.

Holotypus: Kerala, Palghat district, Singampatti to Vattaparai forest, Siruvani western slopes, 625 m, 29.5.1979, E. Vajravelu 62860 (CAL); isotypus (MH).

Allied to C. macrocarpum Hook. f., but differs in having narrow leaves with acute base, closer secondary nerves and oblong fruits. It also differs from $C$. walaiwarense Kosterm. in having glabrous leaves, more closely set up lateral nerves and oblong fruits.

Tree, 12-15 m high (fide collectoris). Branchlets terete, blackish-brown, up to $5 \mathrm{~mm}$ in diameter, axils puberulous, smooth, gradually compressed and quadrangular, densely fine brown puberulous above; leaf-bearing branchlets slender, compressed, densely brown puberulous, 1.5-2.0 mm thick. Terminal buds ovate-lanceolate, c $3 \times 1.5-2.0 \mathrm{~mm}$, acute at apex, densely brown puberulous. Leaves subopposite, thinly coriaceous, greenish-brown above, paler beneath, opaque, narrow oblong-lanceolate to oblongelliptic, $15-22 \times 2.8-4.0 \mathrm{~cm}$, subequally acute at base, slightly decurrent to extreme base, entire, thin, almost flat along margin, apex broken tending to broad acumen; trinerved, central one prominent, raised and stout on both surfaces, square beneath, accessories arising 10-15 mm above from lamina base, raised and stout beneath, gradually slender along length and ultimately vanishing below acumen, lateral nerves of the central one sub-prominent and distant, that of accessories forming faint loops along margin; tertiary nerves parallel, 1-2 mm apart, faint above, obscure beneath; minor nervules faintly reticulate above, obscure beneath; petioles $10-15 \mathrm{~mm}$ long, c $1.5 \mathrm{~mm}$ wide, almost flat with shallow wings along corner above, convex beneath, puberulous. Flowers not seen. Infructescences solitary, axillary, c $15 \mathrm{~cm}$ long; peduncles c $55 \mathrm{~mm}$ long, c $1.5 \mathrm{~mm}$ wide, terete, puberulous; rachis flattened, densely puberulous. Fruits green with large oblong, perianth (fide collectoris) c $22 \times 16 \mathrm{~mm}$, obtuse and projected at apex, pericarp blackish, glabrous, muriculate; fruit cup thick, c $3 \times 12-15 \mathrm{~mm}$, margin slightly undulate, tepal lobes deciduous. Flowering time: Not known. Fruiting time: May-June.

Distribution: India (Kerala).

Habitat: Grows on the hilly slopes at $625 \mathrm{~m}$ altitude. 


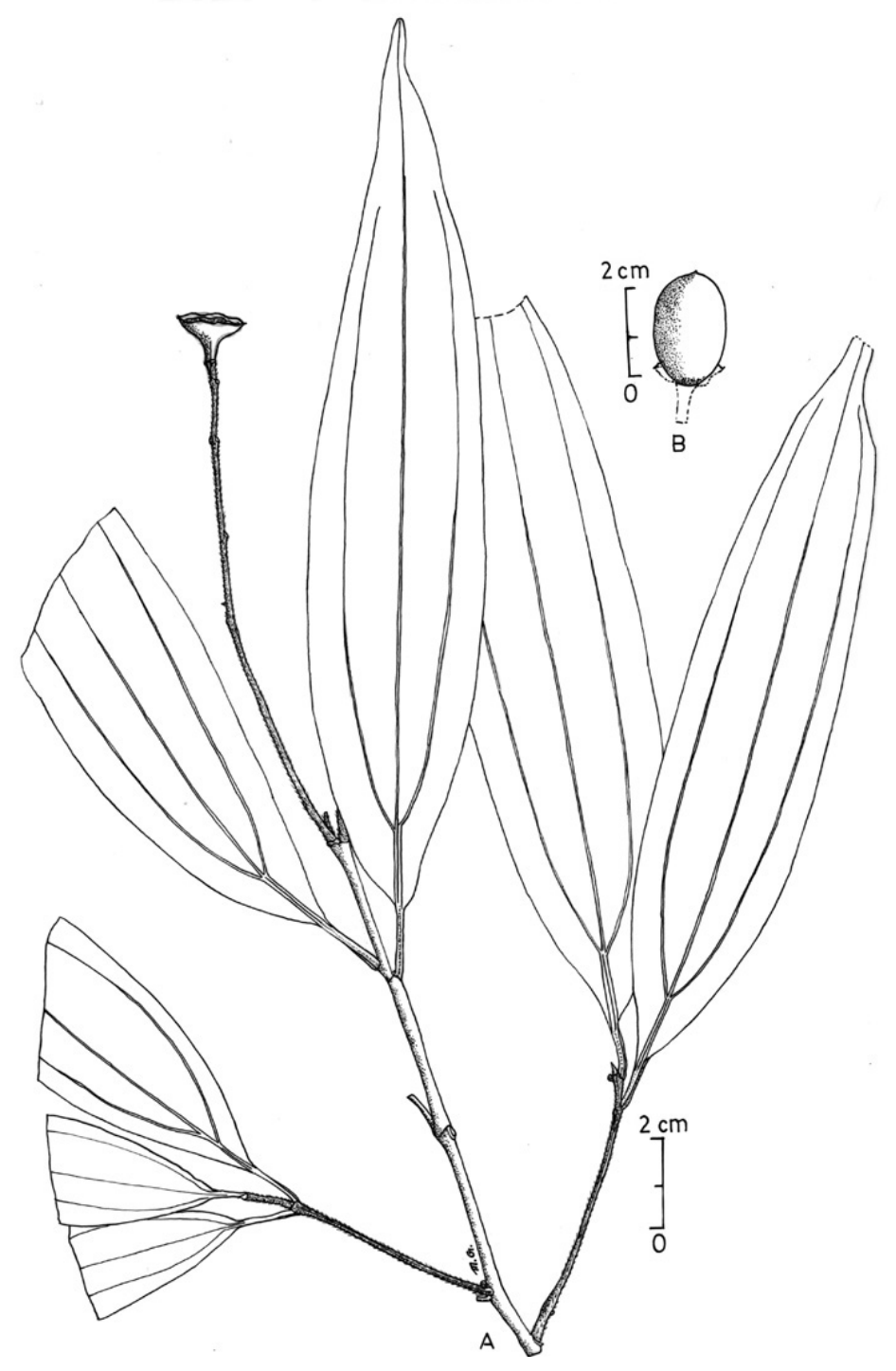

Plate 7. Cinnamomum palghatensis M. Gangop. sp. nov. A. Leafy branch; B. Fruit (after holotype).

8. Cryptocarya calderi M. Gangop., sp. nov.

(Plate 8)

Cryptocarya crassinervia Miq. affinis, sed differt foliis infra cum nervis tertiariis tenuibus, paniculisque laxifloris valde ramosis patentibusque, floribusque subsessilibus.

Holotypus: Upper Cinchona camp, 12.4.1924, C.C. Calder s.n. (CAL).

Allied to C. crassinervia Miq., but differs in having leaves with slender tertiary nerves beneath, lax flowered much branched spreading panicle and subsessile flowers. 
Tree, c $30 \mathrm{~m}$ high. Branchlets terete, longitudinally shallow ribbed and grooved, up to $3.5 \mathrm{~mm}$ in diameter, densely brown tomentose, gradually slender, compressed above. Terminal bud absent. Leaves alternate, coriaceous, green, glossy above, brown, subglaucous beneath, oblong to orbicular-oblong, 13-17 × c $7 \mathrm{~cm}$, unequally obtuse and cuneate, obtuse and obtuse or obtuse and acute at base, non-decurrent to extreme base, strongly incurved along margin, retuse at apex, glabrous except the partially tomentose midvein and lateral nerves above, densely rufus-villous on main nerves and sparsely so elsewhere beneath; midvein slender, channeled above, raised, quadrangular, canaliculate beneath; lateral nerves 5-8 pairs, ascending, arcuate near margin, canaliculate above, raised and stout, forming prominent loops along margin beneath; tertiary nerves faint above, raised and slender beneath, scalariform; minor nervules obscure above, faint beneath, reticulate; petioles to $15 \times 2 \mathrm{~mm}$, flat or shallow concave above, terete beneath, rufus-tomentose. Inflorescences axillary and pseudo-terminal panicles, 6-12 cm long; peduncles 10-35 mm long, c $1.5 \mathrm{~mm}$ in diameter, terete, ferrugineus villous; branches spreading, villous; branchlets slender, flat, villous, lax flowered. Flowers tubularcampanulate, с $4.5 \times 2.8 \mathrm{~mm}$, subsessile, finely appressed tomentellus, bracteolate; pedicels c $0.2 \mathrm{~mm}$ long; bracteoles narrow oblong, acute at apex, ventrally concave, densely tomentellus; tube narrow oblong-obovoid, c $1.5 \times 1.0 \mathrm{~mm}$; tepal lobes $3+3$, acutish at apex, ventrally concave, minutely puberulous within; outer: narrow oblongovate, c $2 \times 1 \mathrm{~mm}$, thick, anastomosis obscure; inner: elliptic-ovate c $2.1 \times 1.2 \mathrm{~mm}$, thin, anastomosis faint. Stamens 9, 3 in each whorl, anthers 2-locular, whorls I and II introrse, of III extrorse, biglandular, filaments puberulous, anthers sparsely puberulous; whorl I: c $1.3 \mathrm{~mm}$ long, filament c $0.5 \mathrm{~mm}$ long, thin; anthers oblong-ellipsoid, obtuse at apex; whorl II: c $1.4 \mathrm{~mm}$ long, filament c $0.7 \mathrm{~mm}$ long, thin; anther ovate-oblong or ovate, subretuse at apex; whorl III: c $1.9 \mathrm{~mm}$ long; gland c $1 \mathrm{~mm}$ long, attached at base of filament; stipes c $0.4 \mathrm{~mm}$ long, thin, puberulous, head sagittate, acute; filament c $1 \mathrm{~mm}$ long, wider below; anther narrow ovate, obtuse at apex; whorl IV (of 3 staminodes): c 1.7 $\mathrm{mm}$ long, stalk short and broad, c $0.3 \mathrm{~mm}$ long, flat, puberulous; head hastate, ventrally shallow grooved, dorsally convex, sparsely puberulous. Pistil glabrescent, c $2.5 \mathrm{~mm}$ long; ovary narrow ovoid, gradually tapering into broad style, c $1 \times 0.5 \mathrm{~mm}$; style flat, shallow channeled; stigma minute, thin, peltate. Fruit not seen. Flowering time: April. Fruiting time: Not known.

Distribution: Myanmar.

Habitat: Grows in the jungle shed.

The species has been named after the well known botanist Mr. C.C. Calder. 


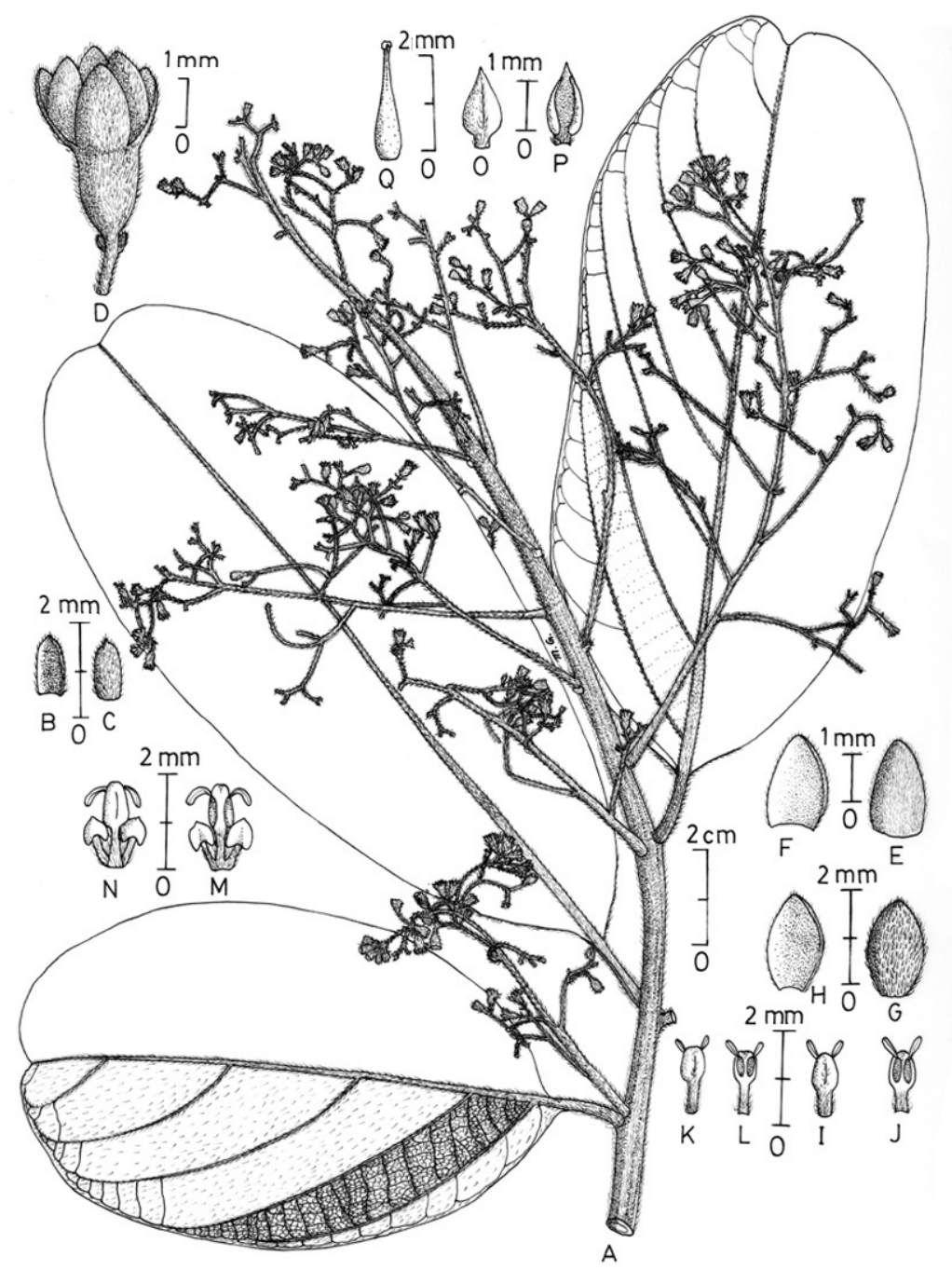

Plate 8. Cryptocarya calderi M. Gangop. sp. nov. A. Flowering branch; B. Bracteole - dorsal view; C. Idem ventral view; D. Flower; E. Outer tepal lobe - dorsal view; F. Idem - ventral view; G. Inner tepal lobe dorsal view; H. Idem - ventral view; I-P. Stamens and staminode: I. Whorl I - dorsal view; J. Idem - ventral view; K. Whorl II - dorsal view; L. Idem - ventral view; M. Whorl III - dorsal view; N. Idem - ventral view; O. Whorl IV - dorsal view; P. Idem - ventral view; Q. Pistil (after holotype).

\section{Cryptocarya simonsii M. Gangop., sp. nov.}

(Plate 9)

Cryptocarya amygdalina Nees affinis, sed differt foliis nitidis supra cum nervulis minoribus obscuris, fructu parvo ovoideo.

Holotypus: India, Assam, Nuku hills, Simons s.n., acc. no. 383079 (CAL); isotypus (K, photo - CAL!). 
Allied to C. amygdalina Nees, but differs in having glossy leaves with obscure minor nervules above and smaller ovoid fruits.

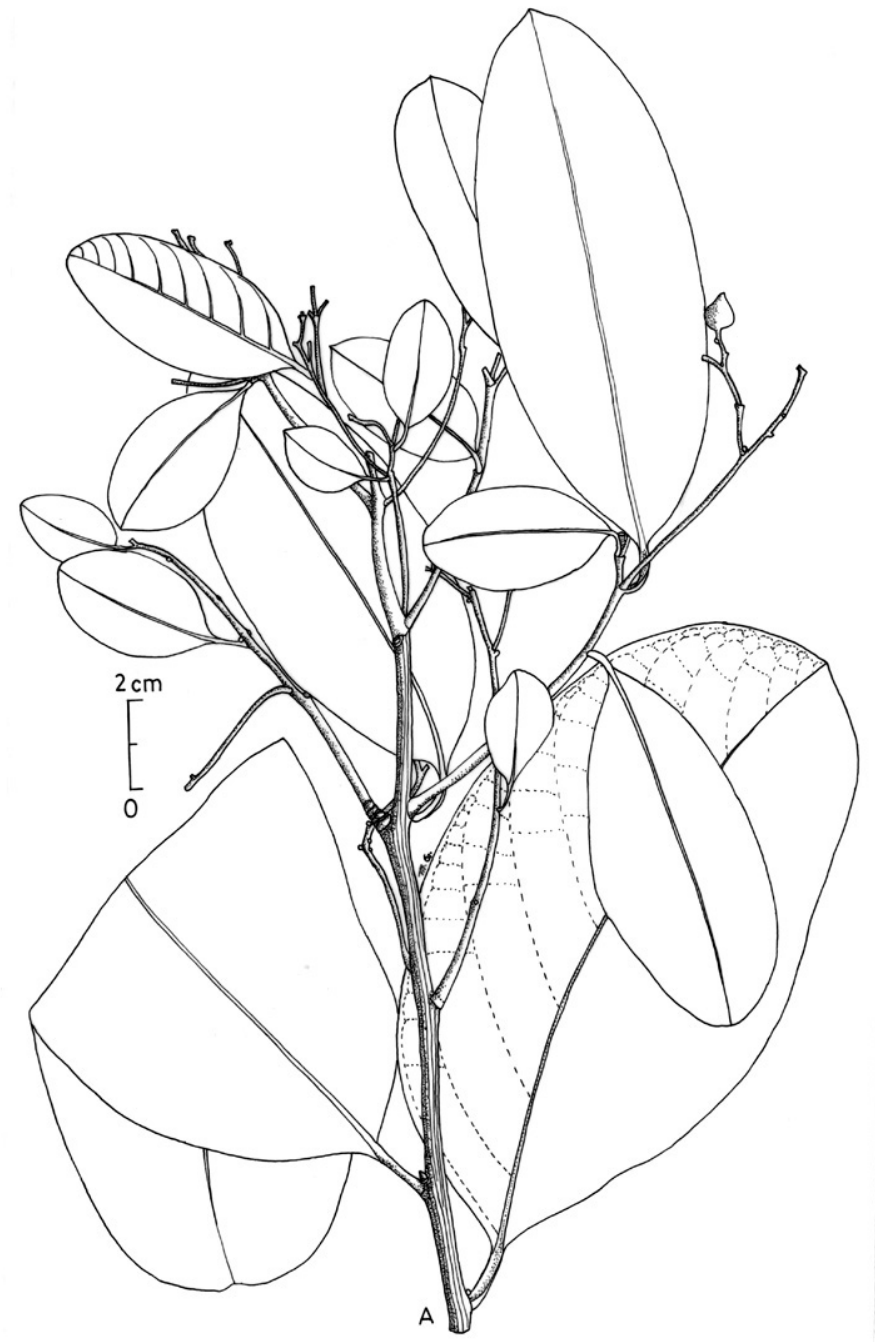

Plate 9. Cryptocarya simonsii M. Gangop. sp. nov. A. Fruiting branch (after holotype).

Branchlets terete, blackish-brown, up to $4 \mathrm{~mm}$ in diameter, longitudinally shallow channeled, sparsely lenticellate, glabrous; gradually slender, compressed, sparsely to densely puberulous above. Terminal buds not seen. Leaves alternate, coriaceous, glossy, greenish-brown above, glaucous beneath, narrow to broad oblong or ovate-oblong, 13-17 $\times 4.5-8.5 \mathrm{~cm}$, equally or subequally obtuse and acute at base, incurved along margin, obtuse and apiculate at apex, glabrous above, appressed pilose beneath when young, glabrous with age; midvein penninerved, shallow channeled above, raised and prominent beneath; lateral nerves 10-12 pairs, slender, ascending, ultimately arcuate near margin, 
flat and faint above, prominent beneath, close, obscurely looped along margin; tertiary nerves faint above, prominent beneath, distantly scalariform; minor nervules obscure above, faint, laxly reticulate beneath; petioles 12-16 mm long, to $2 \mathrm{~mm}$ wide, shallow channeled above, convex beneath, puberulous when young, glabrous with age. Flowers not seen. Infructescences axillary, solitary, up to $8 \mathrm{~cm}$ long; peduncles 38-40 mm long, c $1 \mathrm{~mm}$ wide, glabrescent; branches spreading, slender, glabrescent. Fruits ovoid, c $8 \times 6$ $\mathrm{mm}$, black, smooth, glabrous, tapering above, rounded beneath; stalks c $3 \times 1 \mathrm{~mm}$, sparsely puberulous. Flowering \& Fruiting time: Not known.

Distribution: India (Assam).

Habitat: Grows in the hilly forests.

A duplicate specimen housed at Kew was named by A.J.G.H. Kostermans as $C$. riparia, but he did not publish it.

The species has been named in the honour of Mr. Charles J. Simons, who made extensive collection of plants mainly in the areas of Assam, Khasia and Mikir hills in north-east India.

\section{New combination}

Recently Manickam et al. (2007) have described a new species viz. Beilschmiedia tirunelvelica Manickam et al. from Tamil Nadu, India. But this unique species has been placed erroneously under the genus Beilschmiedia Nees. Beilschmiedia is characterized by trimerous flowers having 9 fertile stamens and 3 staminodes with well-developed filaments as well as inconspicuous decurrent stigma. The description and drawings reveal that $B$. tirunelvelica belongs to the genus Potameia A. Thouars, as it bears dimerous flowers with 4 fertile stamens in two whorls and 2 staminodes as well as inconspicuous stigma.

Thus the following new combination is proposed.

\section{Potameia tirunelvelica (Manickam et al.) M. Gangop., comb. nov.}

Beilschmiedia tirunelvelica Manickam et al. in Nordic J. Bot. 24: 407, fig.1 (2007).

Type: India, Tamil Nadu, Tirunelveli district, Agasthiyamalai Biosphere Reserve, Agasthiar Hills, Poongulam, ca 1400 m, 24.05.1999, V.S. Manickam 19103 (Holotype: $\mathrm{MH}, n . v$.; isotypes: $\mathrm{XCH}, n . v)$.

This species differs from Potameia paradoxa (Hook. f.) Kosterm. (Basionym: Syndiclis paradoxa Hook. f.) of Bhutan in having opposite, oblong-lanceolate leaves with fewer number of lateral veins (5-7), supra-axillary inflorescences and 2-celled anthers. In $P$. paradoxa the leaves are alternate with 10-12 pair lateral veins, the panicles are axillary and the anthers are 1-celled. 
According to Kostermans (1957), the genus Potameia has a disjunctive distribution. So far, 19 are known to occur in Madagascar Island and 1 each in Bhutan and China (Hainan). The occurrence of $P$. tirunelvelica in Tamil Nadu, India is not only a new area of disjunct distribution, but also a new generic record for India.

\section{Acknowledgements}

Thanks are due to the Joint Director and staff members of the Andaman \& Nicobar Circle, Botanical Survey of India, Port Blair for their kind co-operation. Thanks are also

due to Dr. N.C. Majumdar, retired Scientist, Botanical Survey of India for the Latin diagnoses.

\section{References}

Kostermans, A.J.G.H. 1957. Lauraceae. Reinwardtia 4(2): 193-256.

Manickam, V.S., Murugan, C., Jothi, G.J. and Sundaresan, V. 2007. A new species of Beilschmiedia (Lauraceae) from the Western Ghats, India. Nordic J. Bot. 24(4): 407-410. Correction in ibid. 24(6): 703. 2007.

(Manuscript received on 1 April 2008; revised on 15 May 2008) 